\title{
CDC Surveillance Strategy - A Strategy for Improving CDC Activities in Public Health Surveillance
}

\author{
Chesley Richards and Brian Lee* \\ Office of Public Health Scientific Services, CDC, Atlanta, GA, USA
}

\section{Objective}

This presentation aims to share the CDC Surveillance Strategy's goals, initiatives and activities. The surveillance strategy describes how CDC will:

enhance accountability, resource use, workforce and innovation for surveillance by establishing a Surveillance Leadership Board, a surveillance workforce plan, and an innovation consortium;

accelerate the utilization of emerging tools and approaches to improve the availability, quality, and timeliness of surveillance data by establishing enhanced HIT policy engagement, HIT vendor forums, and informatics innovation projects; and

- initiate four cross cutting surveillance system initiatives to improve surveillance by addressing data availability, system usability, redundancies, and incorporation of new information technologies

\section{Introduction}

Public health surveillance guides efforts to detect and monitor disease and injuries, assess the impact of interventions and assist in the management of and recovery from large-scale public health incidents. Today's ever-present, media-hungry environment pressures public health scientists, researchers and frontline practitioners to provide information, on an almost instantaneous basis, responsive to public and policy maker concerns about specific geographies and specific populations. Actions informed by surveillance information take many forms, such as policy changes, new program interventions, public communications and investments in research. Local, state and federal public health professionals, government leaders, public health partners and the public are dependent on high quality, timely and actionable public health surveillance data.

With a charge from the CDC Director, this Surveillance Strategy aims to improve CDC's overall surveillance capabilities, and by extension those of the public health system at large. The Strategy guides efforts to make essential surveillance systems more adaptable to the rapidly changing technology landscape, more versatile in meeting demands for expanding knowledge about evolving threats to health, and more able to meet the demands for timely and populationspecific and geographically specific surveillance information. The Strategy will also facilitate work to consolidate systems, eliminate unnecessary redundancies in reporting, and reduce reporting burden.

These expectations compel this strategy and argue for CDC to lead the public health system in improving the timeliness and availability, as well as the quality and specificity of surveillance data to CDC programs, STLT agencies, and other stakeholders.

\section{Methods}

To improve surveillance efforts at CDC and to make progress on the four strategic goals, CDC will collaborate with principal public health surveillance stakeholders, customers, and partners, and together will focus efforts to achieve three goals to improve CDC health surveillance activities and investments:

Goal 1 - Enhance the accountability, resource use, workforce and innovation for surveillance at CDC and in support of STLT agencies
Goal 2 - Accelerate the utilization of emerging tools and approaches to improve the availability, quality, and timeliness of surveillance data

- Goal 3 - Through cross-cutting agency initiatives, improve surveillance by addressing data availability, system usability, redundancies, and incorporation of new information technologies in major systems or activities

\section{Results}

To improve surveillance efforts at CDC and to make progress on the strategic goals, in 2014 CDC initiated four cross cutting agency initiatives aimed at large surveillance systems:

Initiative 1 - National Notifiable Diseases Surveillance System (NNDSS) Modernization Initiative

- Initiative 2 - BioSense Enhancement Initiative

Initiative 3 - Accelerating Electronic Laboratory Reporting

- Initiative 4 - Mortality Surveillance-Related initiatives with the National Vital Statistics System

\section{Conclusions}

An essential objective and outcome of the board's leadership in implementing these action steps and the entire surveillance strategy will be to optimize CDC's investments in the emerging surveillance systems infrastructure through:

- coordination with STLT health departments, health care providers, and other partners;

transparency in decision making for CDC's surveillance data systems;

- harmonization of CDC's participation in HIT standards development and in developing public health reporting functionality in EHR systems; and

assurance that CDC's future investments in data collection systems - including funding that individual CDC programs provide to STLT health departments - comply with the new surveillance strategy.

\section{Keywords}

surveillance; strategy; workforce; informatics; policy

\section{*Brian Lee}

E-mail: fya1@cdc.gov 